\title{
On a class of Diophantine equations
}

\author{
By Lars Fjellstedt
}

HoRwItz $[1]^{1}$ has studied the Diophantine equation

$$
x_{1}^{2}+x_{2}^{2}+\cdots+x_{n}^{2}=x \cdot x_{1} x_{2} \cdots x_{n} .
$$

For $n=2$ more general equations of this type have been investigated by BARNes [2] and MrLLs [3,4]. This paper deals with the equation

$$
\sum_{i=1}^{n}\left(x_{i}+a_{i}\right)^{2}+c=x \cdot x_{1} x_{2} \cdots x_{n}
$$

where the numbers $a_{i}$ and $c$ are rational integers, $c \geqq 0$. We show that with some alterations the method used by HuRwitz on equation (1) also applies to equation (2). The results obtained are analogous to those of HoRwITz.

We note that if any of the polynomials

$$
\left(x_{i}+a_{i}\right)^{2}+c=0
$$

is reducible, equation (2) is solvable for every value of $x$. Similarly if there exists a solution in which one at least of the numbers $x_{1}, x_{2}, \ldots, x_{n}$ is zero.

In the following lines we consider only solutions in which the numbers $x_{1}, x_{2}, \ldots, x_{n}$ are all $\neq 0$.

Starting from a solution

$$
A=\left(x, x_{1}, x_{2}, \ldots, x_{n}\right)
$$

we can find a new solution $A^{(k)}$ in the following way. In (2) we solve for $x_{k}$. Since the equation considered is of the second degree and since it is satisfied by $x_{k}$, the other root is

$$
x_{k}^{\prime}=x \cdot x_{1} \cdots x_{k-1} x_{k+1} \cdots x_{n}-2 a_{k}-x_{k} .
$$

It is obvious that $x_{k}^{\prime}$ is an integer. Thus

$$
A^{(k)}=\left(x, x_{1}, \ldots, x_{k-1}, x_{k}^{\prime}, x_{k+1}, \ldots, x_{n}\right)
$$

is a solution of (2). The solutions $A^{(k)}, k=1,2, \ldots, n$ are said to be associated with the solution $A$.

1 Numbers in brackets refer to the bibliography at the end of this paper. 


\section{FJELLSTEDT, On a class of Diophantine equations}

A sequence of solutions $A_{1}, A_{2}, \ldots$, of (2) with the property that every solution is associated with the preceding one forms a chain of solutions. It is evident that $x$ has the same value for every solution in a chain.

The number

$$
\sum_{i=1}^{n}\left|x_{i}\right| \quad i=1,2, \ldots, n
$$

is called the weight of the solution $\left(x, x_{1}, \ldots, x_{n}\right)$.

A solution of weight $h$ is said to be fundamental if the weight of all the solutions associated with it is $\geqq h$.

A fundamental solution is said to generate the chain to which it belongs.

We have the following result:

Theorem 1. Every solution of (2)

$$
\left(x, x_{1}, x_{2}, \ldots, x_{n}\right)
$$

with $x_{1}, x_{2}, \ldots, x_{n}$ all $\neq 0$, belongs to a chain generated by a fundamental solution.

Proof. Suppose that a solution $A$ of (2) is not fundamental. Then it has an associated solution whose weight is less than the weight of $A$. If the new solution is not fundamental we can repeat the process. Since the weight is a natural number we must find a fundamental solution after a finite number of steps.

To solve (2) completely we have only to determine the fundamental solutions. Our main result will be that the number of different fundamental solutions is finite and furthermore we will give inequalities for these solutions.

If $\left(x, x_{1}, \ldots, x_{n}\right)$ is a solution of $(2)$ in which the numbers $x_{v_{1}}, x_{v_{2}}, \ldots, x_{v_{r}}$ are negative, $\left(x,\left|x_{1}\right|,\left|x_{2}\right|, \ldots,\left|x_{n}\right|\right)$ is obviously a solution of the equation derived from (2) by changing signs for $a_{v_{1}}, a_{v_{z}}, \ldots, a_{v_{r}}$. Furthermore it is clear that if we can prove for this new equation that the number of different fundamental solutions is finite, the same result holds for (2). Hence we suppose in the sequel that all the numbers $x_{1}, x_{2}, \ldots, x_{n}$ are $>0$.

If the weight of the solution $A$ is not greater than that of $A^{(k)}$, we get from (3) on multiplication with $x_{k}$

$$
x_{k} x_{k}^{\prime}+x_{k}^{2}+2 a_{k} x_{k}=x \prod_{i=1}^{n} x_{i} \text {. }
$$

From (2) we see that $x_{k}$ and $x_{k}^{\prime}$ have the same sign and therefore.

$$
2 x_{k}^{2}+2 a_{k} x_{k} \leqq x \prod_{i=1}^{n} x_{i}
$$

for $k=1,2, \ldots, n$

Let us consider the expression

$$
\begin{aligned}
\left(x \cdot x_{1} x_{2} \cdots x_{k-1} x_{k+1} \cdots x_{n}-2\left(x_{k}+a_{k}\right)\right)^{2}=x^{2} & x_{1}^{2} \cdots x_{k-1}^{2} x_{k+1}^{2} \cdots x_{n}^{2}+ \\
+4 & {\left[x \cdot x_{1} x_{2} \cdots x_{n}\left(1+\frac{a_{k}}{x_{k}}\right)-\left(x_{k}+a_{k}\right)^{2}\right] }
\end{aligned}
$$


which can be written

$$
x \cdot x_{1} x_{2} \cdots x_{n}-2\left(x_{k}^{2}+a_{k} x_{k}\right)=x_{1} x_{2} \cdots x_{n} \sqrt{x^{2}-4 t_{k}}
$$

where

$$
t_{k}=\frac{\left(1+\frac{a_{k}}{x_{k}}\right)\left[\sum_{i=1}^{n}\left(x_{i}+a_{i}\right)^{2}+c\right]-\left(x_{k}+a_{k}\right)^{2}}{x_{1}^{2} x_{2}^{2} \cdots x_{n}^{2}} x_{k}^{2} .
$$

For some index $\mu$ we have

$$
\sqrt{x^{2}-4 t_{\mu}} \leqq \sqrt{x^{2}-4 t_{i}}, \quad i=1,2, \ldots, n .
$$

From (5) we get on addition

$$
n x \prod_{i=1}^{n} x_{i}-2 \sum_{i=1}^{n}\left(x_{i}^{2}+a_{i} x_{i}\right)=\prod_{i=1}^{n} x_{i} \cdot\left(\sum_{i=1}^{n} \sqrt{x^{2}-4 t_{i}}\right),
$$

or

$$
(n-2) x \prod_{i=1}^{n} x_{i}+2 T=\prod_{i=1}^{n} x_{i} \cdot\left(\sum_{i=1}^{n} \sqrt{x^{2}-4 t_{i}}\right),
$$

where

$$
T=\sum_{i=1}^{n}\left(x_{i} a_{i}+a_{i}^{2}\right)+c .
$$

We distinguish two cases according as $T$ is $\leqq 0$ or $>0$.

Suppose first that $T \leqq 0$. Then we get from (8)

$$
(n-2) x \geqq \sum_{i=1}^{n} \sqrt{x^{2}-4 t_{i}} .
$$

As is easily seen, it is sufficient to consider the case $t_{\mu}>0$. In fact, if $t<0$ we must have $t_{i}<0$ for all $i$. It then follows from the left-hand side of (5) that

$$
x_{k}\left(a_{k}+x_{k}\right)<0, \quad k=1,2, \ldots, n .
$$

This, however, means that $x_{k}<\left|a_{k}\right|$ for all $k$.

If $t_{\mu}$ is $=0$, we must have $t_{i} \leqq t_{\mu}$ for all $i$. Thus we get as before $x_{k} \leqq\left|a_{k}\right|$.

On squaring and introducing $t_{\mu}$ instead of $t_{i}$ we get

$$
n^{2} t_{\mu} \geqq(n-1) x^{2}
$$

Substituting in this inequality the expression for $t_{\mu}$ given by (6) we find

$$
n^{2}\left[\left(1+\frac{a_{\mu}}{x_{\mu}}\right)\left(\sum_{i=1}^{n}\left(x_{i}+a_{i}\right)^{2}+c-\left(x_{\mu}+a_{\mu}\right)^{2}\right)\right] \geqq(n-1) \frac{x^{2}}{x_{\mu}^{2}} \prod_{i=1}^{n} x_{i}^{2} .
$$

We now distinguish two cases according as $a_{\mu}$ is $>0$ or $\leqq 0$. If $a_{\mu} \leqq 0$ we get from (9) 
L. FJELLSTEDT, On a class of Diophantine equations

$$
n^{2}\left[\sum_{i \neq \mu}\left(x_{i}+a_{i}\right)^{2}+c\right] \geqq(n-1) \frac{x^{2}}{x_{\mu}^{2}} \prod_{i=1}^{n} x_{i}^{2} .
$$

Suppose now that $\left(x_{m}+a_{m}\right)^{2} \geqq\left(x_{i}+a_{i}\right)^{2}, \quad i \neq \mu$.

Then we have

$$
n^{2}\left[(n-1)\left(a_{m}^{2}+1\right)+c\right] \geqq(n-1) \frac{x^{2} x_{1}^{2} \cdots x_{n}^{2}}{x_{\mu}^{2} x_{m}^{2}} .
$$

If on the other hand $a_{\mu}>0$ we have

Here either

$$
n^{2}\left[(n-1)\left(a_{m}^{2}+1\right) x_{m}^{2}+c+\frac{a_{\mu}}{x_{\mu}} x \prod_{i=1}^{n} x_{i}\right] \geqq(n-1) \frac{x^{2}}{x_{\mu}^{2}} \prod_{i=1}^{n} x_{i}^{2}
$$

$$
x_{\mu} x_{m}^{2} \geqq x \prod_{i=1}^{n} x_{i} \quad \text { or } \quad x_{\mu} x_{m}^{2}<x \prod_{1}^{n} x_{i}
$$

However, in both cases we have the inequality

$$
\prod_{i=1}^{n} x_{i} \cdot \frac{x}{x_{\mu} x_{m}} \leqq \frac{n^{2}}{n-1}\left[(n-1)\left(a_{m}^{2}+1\right)+\left|a_{\mu}\right|+c\right] .
$$

Comparing with (10) we find that if $T \leqq 0,(11)$ is always true.

We turn now to the case $T>0$, and start with a few observations. If $\sum_{i=1}^{n}\left(x_{i}^{2}+a_{i} x_{i}\right)$ is $\leqq 0$, we must have $x_{i} \leqq \sum_{i=1}^{n}\left|a_{i}\right|$. Thus we can assume that this sum is $>0$. From (2) it follows that we have either

$$
\sum_{i=1}^{n}\left(x_{i}^{2}+a_{i} x_{i}\right) \leqq \sum_{i=1}^{n}\left(a_{i} x_{i}+a_{i}^{2}\right)+c
$$

or

If (12) is true

$$
\sum_{i=1}^{n}\left(x_{i}^{2}+a_{i} x_{i}\right)>\sum_{i=1}^{n}\left(a_{i} x_{i}+a_{i}^{2}\right)+c
$$

$$
\sum_{i=1}^{n} x_{i}^{2} \leqq \sum_{i=1}^{n} a_{i}^{2}+c
$$

and thus all the numbers $x_{i}$ are bounded. If on the other hand (13) is true we deduce from (8) and (7)

On squaring we get

$$
(n-1) x \geqq n \sqrt{x^{2}-4 t_{\mu}}
$$

$$
n^{2} t_{\mu} \geqq \frac{2 n-1}{4} x^{2}
$$

We now proceed exactly as in the case $T \leqq 0$. The result is

$$
\frac{x}{x_{\mu} x_{m}} \prod_{i=1}^{n} x_{i} \leqq \frac{4 n^{2}}{2 n-1}\left[(n-1)\left(a_{m}^{2}+1\right)+\left|a_{\mu}\right|+c\right] \text {. }
$$


Combining this inequality with our previous results we have that every fundamental solution of (2) satisfies the inequality

$$
\frac{x}{x_{\mu} x_{m}} \prod_{i=1}^{n} x_{i} \leqq \frac{4 n^{2}}{2 n-1}\left[(n-1) a^{2}+a+n-1+c\right]=C,
$$

where $a=\max \left|a_{i}\right|$.

The following theorem is an immediate consequence of (15):

Theorem 2. The number of different fundamental solutions (chains of solutions) is finite.

(15) gives at once an upper bound for $x$. Furthermore it is possible to determine explicit bounds for all the $x_{i}$. In fact, the following inequality is easily derived from (4):

$$
\left|x^{2}-\left(x_{m}+a_{m}\right)^{2}\right| \leqq a^{2}+a_{m}^{2}+\sum_{i \neq \mu, m}\left(x_{i}+a_{i}\right)^{2}+c .
$$

Since, according to (15), the product

$$
\frac{1}{x_{\mu} x_{m}} \prod_{i=1}^{n} x_{i}
$$

is bounded the same is true for the sum $\sum_{i \neq \mu, m}\left(x_{i}+a_{i}\right)^{2}$.

In fact, we have

$$
\sum_{i \neq \mu, m}\left(x_{i}+a_{i}\right)^{2} \leqq(C+a)^{2}+(n-3)(a+1)^{2} .
$$

Thus we have for $x, x_{i}, i=1,2, \ldots, n$ the inequalities

$$
\left\{\begin{aligned}
x & \leqq C, \\
\left|x_{i}\right| & <\sqrt{(C+a)^{2}+(n-1)(a+1)^{2}+c}+a .
\end{aligned}\right.
$$

Equation (2) with $c<0$ may be treated in the same way as when $c \geqq 0$. However, the details are more complicated. I shall return to this case in a following paper.

\section{REFEREN CES}

1. Horwitz, A., Über eine Aufgabe der unbestimmten Analysis. Math. Werke, Bd. II, S. $410-421$.

2. Barnes, E. S., On the Diophantine equation $x^{2}+y^{2}+c=x y z$. J. London Math. Soc. 28 , 242-244 (1953).

3. MILLs, W. H., A system of quadratic Diophantine equations. Pacific J. of Math. 3, 209-220 (1953).

4. A method for solving certain Diophantine equations. Proc. American Math. Soc. 5 , 473-475 (1954).

Tryckt den 21 april 1955

Uppsala 1955. Almqvist \& Wiksells Boktryckeri AB 